\title{
Capsule Commentary on Weeks et al., "Provider Perceptions of the Electronic Health Record Incentive Programs: A Survey of Eligible Professionals Who Have and Have Not Attested to Meaningful Use"
}

\author{
Jessica S. Ancker, MPH, PhD \\ Department of Healthcare Policy and Research, Division of Health Informatics, Weill Cornell Medical College, New York, NY, USA.
}

J Gen Intern Med 30(1):98

DOI: $10.1007 / \mathrm{s} 11606-014-3045-0$

(c) Society of General Internal Medicine 2014

$\mathrm{W}$ hen Apple released the iPhone 6, customers lined up days in advance. Talking GPS systems are a must-have in new cars. Facebook took only five years to explode from college fad to phenomenon serving more than half of the U.S. population. ${ }^{1}$ Microsoft Office, online-only journals, Twitter, Instagram: Americans love technology.

So why don't doctors love a program that pays them to use technology?

In this month's JGIM, Weeks et. al report high levels of skepticism that the meaningful use program (MU) will accomplish goals such as reducing care disparities or improving accuracy of patient information. ${ }^{2}$ Respondents were concerned about both the reporting requirements and their electronic health records (EHRs), described in open-ended remarks as costly and "cumbersome." Most MU-active providers cited extrinsic motivations for pursuing MU (mandates, incentives, penalties), not intrinsic ones (improved patient care). "I want to remain employed!" one said.

Fifty years ago, sociologist Everett Rogers wondered why some new technologies spread spontaneously while others did not. His "diffusion of innovation" theory ${ }^{3}$ listed predictors on which EHRs still do not always score well. Proficiency requires weeks of training, suggesting lack of simplicity. Providers cannot test-drive EHRs in their own offices before committing (trialability). MU challenges compatibility because, instead of fitting in unobtrusively with social norms about work, it changes workflow and reallocates responsibilities, with the explicit goal of changing medical practice. Many physicians are not convinced that EHRs and MU meet Rogers' criterion of relative advantage over a well-functioning paper record system.
With MU, more U.S. physicians than ever now use EHRs, ${ }^{4}$ but those who are reluctant adopters are unlikely to become enthusiastic ones until improvements to EHRs maximize benefits to physicians. User interfaces must be more intuitive, data capture must be streamlined, and workflow integration must be smoother. It must be simpler to aggregate, analyze, and visualize patient data and best evidence for insights at the point of care. These innovations would minimize the record-keeping burden while maximizing immediate payoffs for physicians, including "patient-centered cognitive support" ${ }^{5}$ for better decision-making and better care.

The mark of success will be the line around the block to greet the latest EHR version upgrade.

Conflict of interest: The author declares that she has no conflict of interest.

Corresponding Author: Jessica S. Ancker, MPH, PhD; Department of Healthcare Policy and Research, Division of Health InformaticsWeill Cornell Medical College, New York, NY, USA (e-mail: jsa7002@med.cornell.edu).

\section{REFERENCES}

1. Horn L. Most Americans have Facebook, study says. PC Magazine 2011.

2. Weeks DL, Keeney BK, Evans PC, Moore QD, Conrad DA. Provider perceptions of electronic health record incentive programs: A survey of eligible professionals who have and have not attested to meaningful use. J Gen Int Med. 2014. doi:10.1007/s11606-014-3008-5.

3. Rogers EM. Diffusion of Innovations. New York: Free Press; 1962/1983.

4. Hsiao C-J, Hing E, Ashman J. Trends in electronic health record system use among office-based physicians: United States, 2007-2013. Nat Health Stat Rep. 2014;75:1-17.

5. Stead WW, Lin HS, editors. Computational Technology for Effective Health Care: Immediate Steps and Strategic Directions. Washington: National Research Council of the National Academies; 2009.

Published online September 30, 2014 